\title{
Uniportal video-assisted thoracoscopic surgery pneumonectomy: an update in the evolution of this minimally invasive approach
}

\author{
William Guido-Guerrero ${ }^{1}$, Diego Gonzalez-Rivas ${ }^{2,3}$ \\ ${ }^{1}$ Department of Thoracic Surgery, Rafael Angel Calderón Guardia Hospital, San José, Costa Rica; ${ }^{2}$ Department of Thoracic Surgery, Coruña \\ University Hospital and Minimally Invasive Thoracic Surgery Unit (UCTMI) Coruña, Coruña, Spain; ${ }^{3}$ Department of Thoracic Surgery, Shanghai \\ Pulmonary Hospital, Tongji University School of Medicine, Shanghai 200433, China \\ Contributions: (I) Conception and design: All authors; (II) Administrative support: All authors; (III) Provision of study materials or patients: All \\ authors; (IV) Data collection and assembly: All authors; (V) Data analysis and interpretation: All authors (VI) Manuscript writing: All authors; \\ (VII) Final approval of manuscript: All authors. \\ Correspondence to: William Guido-Guerrero. Department of Thoracic Surgery, Rafael Angel Calderón Guardia Hospital, San José, Costa Rica. \\ Email: guido.william@gmail.com.
}

\begin{abstract}
Despite the widespread adoption of uniportal video-assisted thoracoscopic surgery (UniVATS) for lung cancer, some procedures are still rarely performed with this approach. A minimally invasive approach is seldom selected to perform a pneumonectomy for a number of different reasons, usually involving the experience of the surgeon and the specific characteristics of cases requiring pneumonectomy as a treatment. Some surgeons believe that because of the complexity of such cases involving large tumors and usually some sort of hilum compromise, a minimally invasive technique cannot be safely performed. Safety involved in performing this procedure is more related to the surgeon experience in the approach than to the procedure itself, and it mostly depends on prior exposure to complex uniportal cases. In a time when several groups around the world are performing bronchovascular reconstructions, carinal resections by UniVATS the question should not be if is possible to perform UniVATS pneumonectomy, but what are the factors and considerations the surgeon must take into account in order to perform these cases. This review aims to provide an update on current status of uniportal VATS pneumonectomy and to provide guidance for surgeons who face this sort of cases.
\end{abstract}

Keywords: Pneumonectomy; minimally invasive; uniportal video-assisted thoracoscopic surgery (UniVATS)

Received: 26 October 2019; Accepted: 19 November 2019; Published: 10 April 2020.

doi: $10.21037 /$ shc. 2019.11 .10

View this article at: http://dx.doi.org/10.21037/shc.2019.11.10

\section{Uniportal video-assisted thoracoscopic surgery (VATS) pneumonectomy: an update}

Thoracic surgery, as any other specialty in medicine, has continuously evolved through time. A lot has changed in the world since Evarts Graham performed the first one stage pneumonectomy for cancer in 1933. At that time, in order to reduce the cavity left by the pneumonectomy, seven ipsilateral ribs were removed, adding considerable morbidity to an already major procedure. Before this successful procedure, pneumonectomies were never performed in a single stage surgery and the morbidity and mortality was prohibitive for this procedure (1). Almost a century has passed by and it has not been in vain. Technology and medical expertise have contributed for this procedure to be performed not only with minimal mortality, but also through a minimally invasive approach with uniportal VATS being one of the last steps in the search for the less invasive effective procedure (2).

For patients with early stages non-small cell lung cancer, the minimally invasive approach in thoracic surgery has included less pain and morbidity, faster recovery and shorter postoperative length of stay $(3,4)$. Similar outcomes in terms of oncologic results have led to the recommendation 
of preferring this approach over open surgery for early stage lung cancer (5). Although we might extrapolate these advantages for surgeries involving lung cancer in advanced stages, there are no prospective randomized trials comparing both approaches for this specific circumstance (6).

Uniportal VATS has been proven to be beneficial and effective for a diverse range of complex major pulmonary resections, including centrally located tumors, post induction cases, bronchovascular reconstructions and carinal resections (7).

The most important thing to keep in mind is the Uniportal VATS is a minimally invasive approach designed to achieve a complete oncologic resection with minimal physical trauma to the patient, without compromising oncologic principles. For that reason, the indication for a pneumonectomy has nothing to do with the surgical approach selected for the patient, but with the staging and physical status of the patient.

\section{Indications}

Parenchymal sparring procedures are always preferred when operating a patient with a centrally located cancer, but in some cases, there is involvement on the distal part of the other lobes, making a pneumonectomy necessary in order for the procedure to be curative (8). The instances in which a VATS pneumonectomy should be performed include:

- Centrally located tumors not amenable to sleeve resections;

- Fissure tumors involving vascular and bronchial structures of all the ipsilateral lobes;

- Synchronous ipsilateral tumors involving multiple lobes.

One of the main prerequisites for being able to perform a UniVATS pneumonectomy is prior experience and expertise in UniVATS major lung resections; the complexity of these cases demand expertise handling difficult hilum in order to prevent possible complications. There are some special considerations that are unique in order to consider a patient suitable for a VATS approach for a pneumonectomy, including the uniportal approach. First, the patient must be able to tolerate single lung ventilation, because without this, instrumentation and visualization would be almost impossible. Second, the size of the tumor, as big tumors measuring more than $7 \mathrm{~cm}$ in diameter can be very difficult to handle by uniportal VATS and will probably require some sort of thoracotomy to extract the lung. Even so, the authors believe that in big tumors, trauma to the intercostal nerve can be minimized if the procedure is completed by VATS and the incision is enlarged at the end. If still necessary, a rib spreader can be used momentarily to achieve enough space to remove the specimen instead of maintaining it during the whole procedure when a thoracotomy is performed, keeping the nerve compressed for a longer time. Considerable experience in UniVATS is necessary to successfully complete these challenging cases.

\section{Is it feasible and safe to perform a uniportal VATS pneumonectomy?}

Subxiphoid and uniportal VATS are both the most recent steps in the evolution of minimally invasive thoracic surgery. UniVATS for major lung resections have only been around since 2011 (9) and for this reason, despite the widespread adoption of the technique around the world, many of the teams doing UniVATS major pulmonary resections are still obtaining experience with early stage lung cancer before feeling comfortable enough with the approach to perform advance tumor cases. Although technically speaking, dissecting and ligating the pulmonary vessels at the hilum should not be a factor that increases the difficulty of the procedure by itself, cases that require a pneumonectomy have special characteristics that could make this process a complex task. First of all, tumors tend to be centrally located in the hilum, distorting the anatomy and, in some cases, even infiltrating the vessels or bronchus at a central level, making very difficult to find a dissection plane to safely isolate the structure, sometimes requiring that the pericardium be opened in order to complete the task. Second, these cases usually tend to be advance stages of lung cancer, amenable to induction chemoradiotherapy, and performing a minimally invasive radical pneumonectomy after induction therapy can be quite challenging due to fibrosis, adhesions and inflammation in the pulmonary hilum (10).

Conventional VATS pneumonectomy has been found to be feasible and effective by many groups performing this surgery (11-13), and probably, with enough time, the same results will be found for UniVATS.

Despite how new this technique is, already some groups around the world have shown good results with the UniVATS approach for pneumonectomies. The largest series to date reported 10 cases in 2013 (14), but other groups have also replicated this results with the UniVATS technique $(15,16)$.

Given the foregoing, with enough experience and an 
appropriate learning curve, UniVATS is a feasible surgical approach for pneumonectomy in which the limitation of the approach for especially challenging cases, such as those after induction therapy, will be dictated mainly by the experience of the surgeon with the technique instead of the specific procedure by itself.

\section{Does uniportal VATS provide a benefit for these cases?}

The one thing to keep in mind is that the goal of achieving a UniVATS Pneumonectomy is to provide the patient all the benefits of a minimally invasive approach, without compromising the safety of the patient and the oncologic results of the operation. There are no prospective studies that compare UniVATS pneumonectomy to the open approach and probably it will be some time before enough groups are performing this surgery in order to be able to truly compare both techniques and their long-term results. But, if we keep in mind that all the oncologic principles have to be preserved with the approach (complete lymph node dissection, negative margins, individual hilar dissection and stapling of the bronchovascular structures) the results obtained should not differ from an open approach. Some groups have already reported similar results in terms of radical resection in pneumonectomy between conventional VATS and open surgery (12). One important thing is that all of these criteria can be monitored by the surgeon performing the surgery and, if the surgeon is not able to comply with them, a change in approach must be made during the surgery. If the surgeon is not able to perform a complete lymphadenectomy because of lack of experience or technical issues than he/she should considered converting to an open procedure. The same applies for an appropriate margin of resection, short bronchial stump, etc.

Adding to the expected similar oncologic results patients have the potential advantages obtain with a minimally invasive approach, less trauma, less pain and shorter in hospital stay, faster administration of adjuvant therapy. However, these advantages have not been as clear for pneumonectomy as they have shown to be for VATS lobectomy (17).

One thing to keep in mind is that the physiological impact of a pneumonectomy is greater than the one that results from a lobectomy, so the difference in postoperative complication rates may not differ as much between an open and a minimally invasive approach. As shown in the study of Liu et al, lower postoperative pain was documented, but no statistical difference in postoperative complications (arrhythmias, bleeding, pneumoniae) (12) was observed.

Taking these factors into account, reducing the surgical trauma of a thoracotomy in an already significantly major procedure such as a pneumonectomy could aid the patient in the recovery process and diminish the affection of the acute postoperative period. Prospective randomized studies are necessary to document the real impact of the approach in the postoperative evolution.

\section{Technical considerations}

There are general considerations that apply for the right and left pneumonectomy. Patients should be positioned on lateral decubitus according to the side that is going to be operated. Using the mid axillary line as reference, a $4-5 \mathrm{~cm}$ incision is usually made in the $5^{\text {th }}$ intercostal space, although some surgeons prefer the $4^{\text {th }}$ intercostal space for a right pneumonectomy (sometimes it is necessary to enlarge the incision in order to extract the specimen). The manner in which the anterior or posterior incision is made differs according to the surgeon's preference and anatomical considerations for a specific patient.

A $30^{\circ}$ camera is placed in the posterior part of the wound and remains there during the whole procedure. This is of upmost importance because the camera and the instruments must mimic the position of the eyes and hands of the surgeon, as in open surgery, so instrumentation becomes easier and more fluid. Specific uniportal VATS instruments are not mandatory to successfully complete the procedure but are extremely useful and considerably facilitate the surgery. The assistant can be on the same side of the table or in front. For experienced and seasoned teams in uniportal VATS, the assistant is located in the contralateral side of the surgeon, which is more comfortable for the surgeon since it provides more space to work in and prevents "fighting" during instrumentation, but is more demanding for the assistant holding the camera. Specific circumstances for each side are as follows:

\section{Right pneumonectomy}

The procedure usually starts with the dissection of the vascular structures at the hilum, that are facilitated by retracting the lung downwards for the dissection of the 


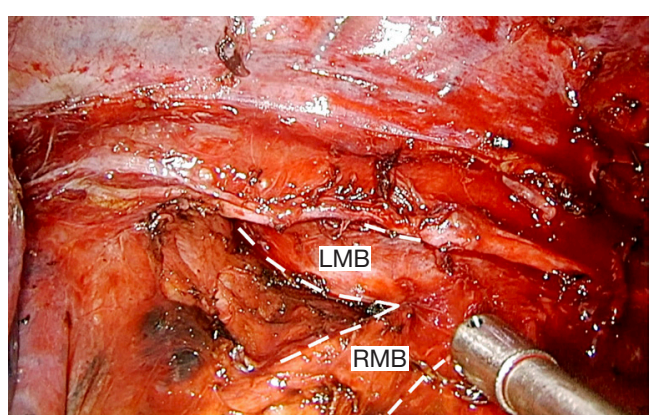

Figure 1 Station 7 lymph node dissection carried out before stapling the bronchus, especially in the right side will allow a better exposure of the carina and contribute to a shorter bronchial stump. $\mathrm{RMB}$, right main bronchus; $\mathrm{LMB}$, left main bronchus.

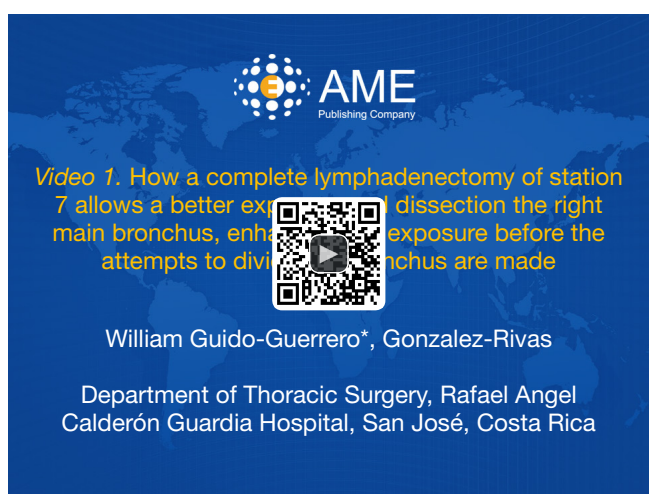

Figure 2 This video shows how a complete lymphadenectomy of station 7 allows a better exposure and dissection the right main bronchus, enhancing the exposure before the attempts to divide the bronchus are made (20).

Available online: http://www.asvide.com/watch/33071

pulmonary artery, upper and middle lobe veins and upwards for the lower lobe vein (18). Bimanual instrumentation is very important in order to keep adequate traction and to facilitate dissection (19). First, for dissection of the main pulmonary artery, the authors recommend stapling the anterior trunk, which provides more space resulting in a better angle to pass the stapler through the upper lobe vein. Another option is to do dissection of the upper pulmonary vein first, but this requires retracting the upper lobe anteriorly and to the apex, in order to prevent injury to pulmonary artery with the stapler. The surgery continues with the dissection of the lower pulmonary vein, starting with the ligament and doing a meticulous dissection of the posterior part of the vein, removing all tissue between the vein and the bronchus, so encircling the vein becomes quite easy and considerably facilitates passing the stapler. The next step is dissection of the pulmonary artery. At this time, since both veins have been transected, visualization of the PA is enhanced, so it allows a safer dissection of the structure. Station 10 lymph nodes are recommended to be dissected first, enhancing the exposure. Careful dissection of the posterior part of the PA is important to facilitate isolation of the artery with a right angle. Silk or rubber bands can be used to aid in the retraction and provide more space for the stapler to pass. The main bronchus is then dissected away from lymph nodes and peribronchial tissue to allow good retraction and a safe oncological margin with a short bronchial stump after stapling. The foregoing is to prevent possible complications, such as bronchopleural fistula (18). A helpful tip is to perform station 7 lymphadenectomy prior to the stapling and division of the bronchus (Figure 1), since it allows more space, mobility and exposure to divide the right main bronchus (Figure 2).

A complete lymph node dissection is performed afterwards, including in the right-side station 2-4r, 10, 7, 8 and 9 (if it has not been performed before).

\section{Left pneumonectomy}

In the left side, dissection starts with the upper part of the hilum, between the left PA and the upper lobe vein, with a thorough dissection between both structures. It is very important to dissect as centrally and distally possible in all the surface of the vessel, so it becomes quite free and mobile (Figure 3). Dissection proceeds with the posterior cranial part of the left PA and lymph node dissection of the aortopulmonary window, which is better to be performed at this moment so it provides adequate exposure of the posterior part of the $\mathrm{PA}$ to pass a right angle to isolate the structure. A rubber band can be used to aid with the retraction and exposure of the left PA to facilitate passing of the stapler or the sucker can be used as a guide for the stapler to pass between the left superior pulmonary vein and the pulmonary artery (Figure 4). The next step is to staple the left upper vein, which is already dissected from the previous step, and finally dissection of the lower lobe vein following the pulmonary ligament. The bronchus should be dissected as proximally as possible to ensure a short stump, which is especially important in the left side since the left 


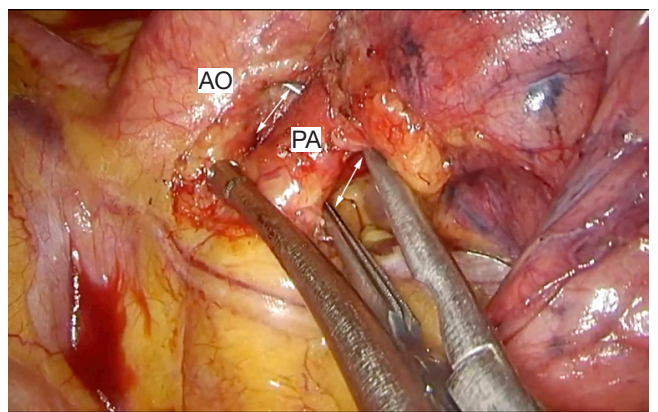

Figure 3 During the hilum approach, meticulous dissection around the vessel, as distally and proximally as possible (illustrated with white arrows)will simplify considerably the stapler passage across the vessel. PA, pulmonary artery; Ao, aorta.

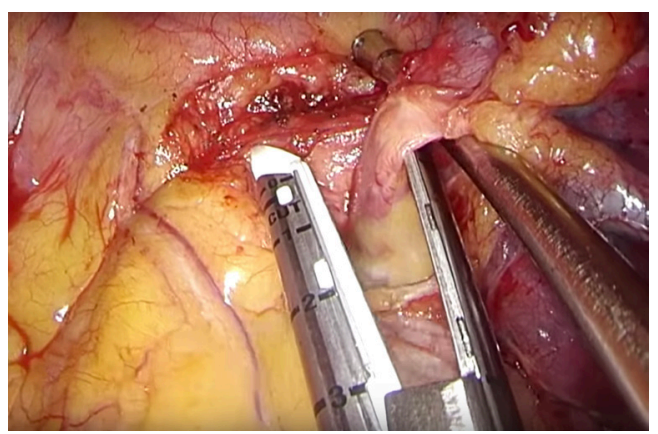

Figure 4 This picture shows how to place the sucker between the pulmonary artery and vein in order to guide the stapler when is passed below the vein reducing the risk of injuring the pulmonary artery behind.

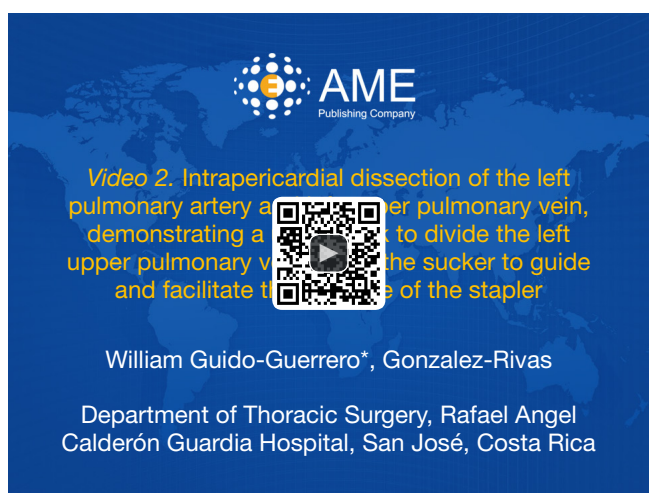

Figure 5 Intrapericardial dissection of the left pulmonary artery and left upper pulmonary vein, demonstrating a useful trick to divide the left upper pulmonary vein, using the sucker to guide and facilitate the passage of the stapler (21).

Available online: http://www.asvide.com/watch/33072 bronchus anatomically is staple further from the carina. Then, the procedure proceeds with a mediastinal lymph node dissection of station $10,5,7,8$ and 9 .

\section{Intrapericardial pneumonectomy}

It is not infrequent that tumors in need of a pneumonectomy for their resection infiltrate the vessels so proximal into the hilum that a dissection plane cannot be found at the extra pericardial level or a negative resection margin is not possible unless an intrapericardial dissection of the vessels is performed.

An intrapericardial pneumonectomy is a more complex procedure as it requires precision and skill to open de pericardium and surround the PA and both pulmonary veins without injuring the phrenic nerve and pushing or compressing the heart excessively (Figure 5). But, even taking this into consideration, it is less difficult and dangerous than trying to force a dissection plane through a tumor infiltrated pulmonary artery or hilar infiltrated lymph nodes.

The pericardium is opened posterior to the phrenic nerve, usually with the hook cautery, but this can also be done with scissors (Figure 6). Retracting the pericardium away from the heart with a grasper facilitates maneuvering and avoids accidental injuries to the heart (Figure 7). The dissection is than continued through the pericardium alongside the vessels to be able to encircle them. It is important to be aware of adhesions or fibrosis between the heart and the inner layer of the pericardium so extra care is taken to avoid injuries. After the dissection is completed, a vessel loop can be used to facilitate passing of the stapler, but it is not always necessary. To improve the angles for introducing the stapler for the PA and the upper lobe vein, the lung is retracted caudally and laterally, and for the lower lobe vein cranially and laterally.

During dissection of the PA in left pneumonectomy, it is especially important to be aware of the left recurrent laryngeal nerve, so inadvertent injury can be avoided.

\section{Take home message}

Although this is a procedure every thoracic surgeon tries to avoid, pneumonectomy is still necessary to resect advance stage lung cancers and cannot be underestimated. Cases in which pneumonectomy is the only surgical option for treatment usually involve large tumors, complex and infiltrated hilum and challenging dissection involving 


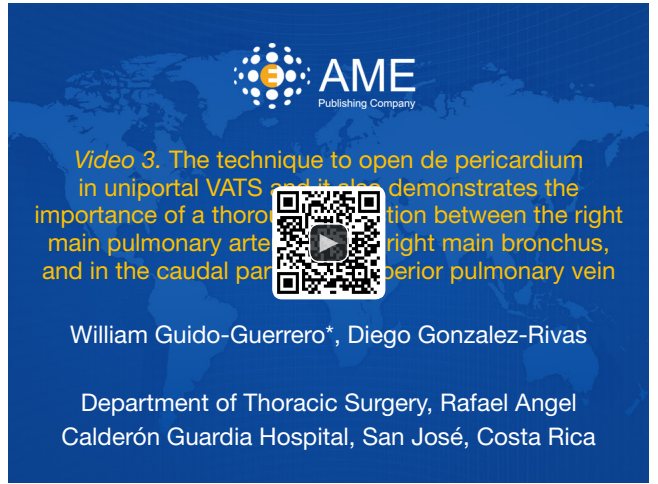

Figure 6 This video shows the technique to open de pericardium in uniportal VATS and it also demonstrates the importance of a thorough dissection between the right main pulmonary artery and the right main bronchus, and in the caudal part of the superior pulmonary vein. Time invested in this part will facilitate considerably the encircling and the stapler passage between the vessels (22). VATS, video-assisted thoracic surgery.

Available online: http://www.asvide.com/watch/33073

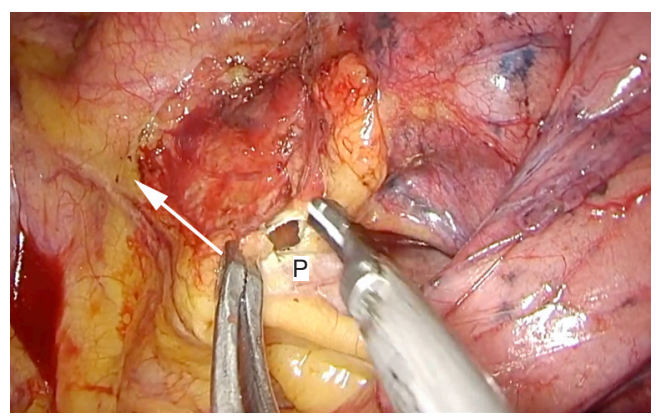

Figure 7 This picture shows one of the maneuvers to open pericardium $(\mathrm{P})$. The pericardium is grasp laterally to the phrenic nerve and traction is apply medially and upwards (white arrow). Scissors, ultrasonic or bipolar devices can be used to open the pericardium medially to the instrument grasping it.

enlarge lymph nodes. To perform these cases with a minimally invasive approach, such as uniportal VATS, is feasible, but expertise is mandatory to be able to handle complex hilum and, in some necessary cases, to perform an intrapericardial pneumonectomy. Although the advantages of a minimally invasive approach may not be as apparent as in lobectomies because the physiological consequences of a pneumonectomy are still present.

Thoracic surgeons should add this approach to their skill set in order to minimize the physical trauma related to the surgery. Uniportal VATS still has a long road to cover, but for now, is a feasible option to operate these advance cases as long as the surgeon is experienced with the technique.

\section{Acknowledgments}

None.

\section{Footnote}

Conflicts of Interest: The authors have no conflicts of interest to declare.

Ethical Statement: The authors are accountable for all aspects of the work in ensuring that questions related to the accuracy or integrity of any part of the work are appropriately investigated and resolved.

\section{References}

1. Fell SC. Special article: a brief history of pneumonectomy. 1999. Chest Surg Clin N Am 2002;12:541-63.

2. Gonzalez-Rivas D. VATS lobectomy: surgical evolution from conventional VATS to uniportal approach. ScientificWorldJournal 2012;2012:780842.

3. Bendixen M, Jørgensen OD, Kronborg C, et al. Postoperative pain and quality of life after lobectomy via video-assisted thoracoscopic surgery or anterolateral thoracotomy for early stage lung cancer: a randomized controlled trial. Lancet Oncol 2016;17:836-44.

4. Villamizar NR, Darrabie MD, Burfeind WR, et al. Thoracoscopic lobectomy is associated with lower morbidity compared with thoracotomy. J Thorac Cardiovasc Surg 2009;138:419-25.

5. Detterbeck FC, Lewis SZ, Diekemper R, et al. Diagnosis and Management of Lung Cancer, 3rd ed. Chest EvidenceBased Clinical Practice Guidelines.

6. Nagai S, Imanishi N, Matsuoka T, et al. Video-assisted thoracoscopic pneumonectomy: retrospective outcome analysis of 47 consecutive patients. Ann Thorac Surg 2014;97:1908-13.

7. Huang J, Li J, Qiu Y, et al. Thoracoscopic double sleeve lobectomy in 13 patients: a series report from multicenters. J Thorac Dis 2015;7:834-42.

8. Abdelsattar ZM, Shen KR, Yendamuri S, et al. Outcomes After Sleeve Lung Resections Versus Pneumonectomy in the United States. Ann Thorac Surg 2017;104:1656-64. 
9. Gonzalez D, Paradela M, Garcia J, et al. Single-port videoassisted thoracoscopic lobectomy. Interact Cardiovasc Thorac Surg 2011;12:514-5.

10. White A, Kucukak S, Bueno R, et al. Pneumonectomy is safe and effective for non-small cell lung cancer following induction therapy. J Thorac Dis 2017;9:4447-53.

11. Yang CJ, Yendamuri S, Mayne NR, et al. The role of thoracoscopic pneumonectomy in the management of non-small cell lung cancer: A multicenter study. J Thorac Cardiovasc Surg 2019;158:252-264.e2.

12. Liu Y, Gao Y, Zhang H, et al. Video-assisted versus conventional thoracotomy pneumonectomy: a comparison of perioperative outcomes and short-term measures of convalescence. J Thorac Dis 2016;8:3537-42.

13. Sahai RK, Nwogu CE, Yendamuri S, et al. Is thoracoscopic pneumonectomy safe? Ann Thorac Surg 2009;88:1086-92.

14. Gonzalez-Rivas D, Delgado M, Fieira E, et al. Uniportal video-assisted thoracoscopic pneumonectomy. J Thorac Dis 2013;5 Suppl 3:S246-252.

15. Halezero-lu S. Single incision video-assisted thoracic surgery pneumonectomy for centrally located lung cancer. Future Oncol 2018;14:41-5.

16. Vannucci F, Vieira A, Ugalde PA. The technique of VATS right pneumonectomy. J Vis Surg 2018;4:11.

17. Battoo A, Jahan A, Yang Z, et al. Thoracoscopic pneumonectomy: an 11-year experience. Chest

doi: 10.21037/shc.2019.11.10

Cite this article as: Guido-Guerrero W, GonzalezRivas D. Uniportal video-assisted thoracoscopic surgery pneumonectomy: an update in the evolution of this minimally invasive approach. Shanghai Chest 2020;4:21.
2014;146:1300-9.

18. Gonzalez-Rivas D, Sihoe ADL. Important technical details during uniportal video-assisted thoracoscopic major resections. Thorac Surg Clin 2017;27:357-72.

19. Gonzalez-Rivas D. Unisurgeon' uniportal video-assisted thoracoscopic surgery lobectomy. J Vis Surg 2017;3:163.

20. Guido-Guerrero W, Gonzalez-Rivas D. How a complete lymphadenectomy of station 7 allows a better exposure and dissection the right main bronchus, enhancing the exposure before the attempts to divide the bronchus are made. Asvide 2020;7:031. Available online: http://www.asvide.com/ watch/33071

21. Guido-Guerrero W, Gonzalez-Rivas D. Intrapericardial dissection of the left pulmonary artery and left upper pulmonary vein, demonstrating a useful trick to divide the left upper pulmonary vein, using the sucker to guide and facilitate the passage of the stapler. Asvide 2020;7:032. Available online: http://www.asvide.com/watch/33072

22. Guido-Guerrero W, Gonzalez-Rivas D. The technique to open de pericardium in uniportal VATS and it also demonstrates the importance of a thorough dissection between the right main pulmonary artery and the right main bronchus, and in the caudal part of the superior pulmonary vein. Asvide 2020;7:033. Available online: http://www.asvide.com/watch/33073 